\title{
Designing Sustainable Smart Connected Communities using Dynamic Spectrum Access via Band Selection
}

\author{
Vijay K. Shah \\ University of Kentucky, Lexington, USA \\ vijay.shah@uky.edu \\ Simone Silvestri \\ University of Kentucky, Lexington, USA \\ silvestri@cs.uky.edu
}

\begin{abstract}
While designing communication systems for sustainable smart connected communities (SCCs), energy efficiency is as important as quality of service (QoS). However, simultaneous achievement of both is difficult due to the varying demands posed by heterogeneous sensing modalities, lack of dedicated infrastructure in rural areas, and certain sustainability constraints. While low-power short range technologies often fail to achieve high QoS, using 3GPP technologies (LTE, LTE-A,GSM) for SCCs will eventually face spectrum scarcity and cross technology interference. While dynamic spectrum access (DSA) has been proposed to overcome policy constraints and improve spectrum scarcity by spectrum sharing, we show in this paper that harnessing DSA in the context of SCCs can achieve benefits in terms of end-to-end energy efficiency. This paper proposes a novel architecture for SCCs using a small scale DSA enabled overlay network over legacy infrastructure to improve the endto-end energy efficiency while guaranteeing QoS. Specifically, our approach selectively exploits distinct electro-magnetic characteristics of various bands in order to intelligently match any message requirement with a suitable band, and hence determines the optimal TTL constrained energy-efficient (TcE) path that enhances the end-to-end energy efficiency and meets the TTL deadline. We formulate a constrained optimization problem and propose a dynamic programming approach for the determination of optimal TcE path for any given message. Compared to the homogeneous band approaches that opportunistically accesses channels within a predetermined band, our studies show that the band selection approach improves the energy efficiency by almost $40 \%$ while preserving the QoS.
\end{abstract}

\section{CCS CONCEPTS}

- Networks $\rightarrow$ Cognitive radios; • Hardware $\rightarrow$ Energy distribution; Impact on the environment; Sensor applications and deployments; Emerging technologies; • Computer systems organization $\rightarrow$ Embedded and cyber-physical systems;

Permission to make digital or hard copies of all or part of this work for personal or classroom use is granted without fee provided that copies are not made or distributed for profit or commercial advantage and that copies bear this notice and the full citation on the first page. Copyrights for components of this work owned by others than ACM must be honored. Abstracting with credit is permitted. To copy otherwise, or republish, to post on servers or to redistribute to lists, requires prior specific permission and/or a fee. Request permissions from permissions@acm.org.

BuildSys'17, November 8-9, 2017, Delft, The Netherlands

(C) 2017 Association for Computing Machinery.

ACM ISBN 978-1-4503-5544-5/17/11 \$15.00

https://doi.org/10.1145/3137133.3137153

\author{
Shameek Bhattacharjee \\ Missouri University of Science and Technology, Rolla, USA \\ shameek@mst.edu \\ Sajal K. Das \\ Missouri University of Science and Technology, Rolla, USA \\ sdas@mst.edu
}

\section{KEYWORDS}

Dynamic Spectrum Access; Energy Efficiency; Band Selection

ACM Reference Format:

Vijay K. Shah, Shameek Bhattacharjee, Simone Silvestri, and Sajal K. Das. 2017. Designing Sustainable Smart Connected Communities using Dynamic Spectrum Access via Band Selection. In Proceedings of 4th ACM Conference on Systems for Energy-Efficient Built Environments (BuildSys'17). ACM, New York, NY, USA, 10 pages. https://doi.org/10.1145/3137133.3137153

\section{INTRODUCTION}

Ubiquitous connectivity is a crucial requirement for future smart and connected communities (SCCs) whether rural, sub-urban or urban. Such connectivity enables to gather, disseminate, and analyze information on multiple sensing modalities, for efficient decision making on several frontiers of human life [34]. To form such SCCs, huge communication infrastructure is needed to offload data from millions of physical sensor blocks to their respective decision making units (e.g., a data center) usually within certain time deadlines (a QoS metric). Such communities may be formed in both urban and non-urban areas. For example, a sensor block could be a remote rural farm equipped with moisture sensors, soil monitors, and wind sensors; or a smart home with temperature, light sensor, and power consumption meters; whose data needs to be offloaded to a central utility for decision making on various purposes.

However, realization of huge communication infrastructure faces economic and sustainability hurdles for both non-urban and urban areas around the world. First, it is not cost-effective for providers to invest in dedicated infrastructure like base stations, access points with backhaul links, spectrum licenses in non urban areas due to low revenue returns compared to the high initial investment [3], [12]. Hence, sensor blocks planned in non-urban areas are usually far away from the data center or the nearest backhaul link. Second, while certain real time latency critical decisions having a local area scope may use fog computing [5] (which requires dedicated infrastructure), many decisions need data over a wider area over long term. Therefore, even in urban areas, the data needs to travel larger distances between data center and the sensor blocks. Even if the existence of infrastructure is assumed (as in urban areas), sending data collected from millions of such sensor blocks are likely to burden the existing wired networks and in turn require deployment of more routers, switches, which would need additional power and hence, exacerbates the problem of energy sustainability.

To address the difficulties related to infrastructure costs (rural/suburban areas) and burdening the wired network (urban areas), SCCs 
could be achieved through a network of fixed (or mobile) cyber physical systems (e.g., road side units (RSUs), private smart vehicles, public transport) connected through wireless links [7], [35], without needing additional dedicated infrastructures. Such an option is feasible for decisions in SCCs that do not have a very short real-time deadline (also, termed as time-to-live (TTL)). E.g., in case of SCC such as a rural smart home constituting smart meters and indoor air quality, humidity etc. A smart meter data usually have 1 hour TTL for billing cycle whereas other basic sensor data may tolerate higher time delays, like 5-6 hours. Some generic ideas pertaining to such a possibility has been discussed in [34]. Therefore, wireless spectrum becomes a critical resource for the success of SCCs.

Current wireless technologies for SCCs typically include (i) Low Power Short Range (e.g. ZigBee, Bluetooth, WiFi, Low6PAN using $2.4 \mathrm{GHz}$ ) and (ii) 3GPP Standards (e.g., LTE, GSM, LTE-A using $1700-2100,900 \mathrm{MHz}$ ). Low power technologies though seemingly energy-efficient, have a limited transmission coverage [11]. Since, the data often needs to travel larger distances, a large number of devices need to be deployed to form a multi-hop network [19] such that a high QoS (data offloading within its TTL deadline) is guaranteed. However, such a communication network exacerbates the problem of both energy efficiency and monetary cost.

On the other hand, though 3GPP technologies (dominant in urban areas) provide higher transmission coverage, they come at a cost of higher energy expenditure [16] due to two major reasons. First, the co-existence of increasingly myriad devices in the same spectrum will cause cross technology interference negatively impacting the SINR requirement. Therefore, either increased power or more re-transmissions would be required (see Sec 4). Second, the homogeneity of legacy spectrum access policy does not provide the flexibility to handle data traffic with heterogeneous demands (such as varying message sizes and TTL deadlines) that may be generated from the same sensor block. Such homogeneity of the spectrum access plan triggers the disadvantage of resource over-provisioning or under-provisioning that again, negatively impacts the achievable energy efficiency (see Sec 4).

In the last decade, Dynamic Spectrum Access (DSA) [2] has emerged as an enabling paradigm that allows certain wireless devices to opportunistically switch and access two or more unoccupied bands (called whitespaces and grayspaces) originally licensed for other services on the condition of non-interference to the primary licensee (or primary users). This provides an option to break away from the homogeneity of legacy spectrum access without the provider having to buy a fixed spectrum license. While the original purpose of DSA was to improve spectrum efficiency [30] and reduce cross technology interference, the fact that it allows switching among different bands could be used to improve the overall energy efficiency by opportunistically matching the electromagnetic properties of each band with the requirements of different sensing modalities being sensed in the SCC. In fact, many 5GPP working committees are exploring various bands apart from the traditional fixed access. Till date, whitespace and grayspace networking has been allowed by policy in TV Band [22], [17], [15], GSM Band [12], LTE Band [33], [32], and Citizen Broadband Radio Service (CBRS) Band [20]. Empty channels within each band are liable to be selected for the actual communication. However, a decade long body of research has been focused on interference free channel assignment given a particular band [6], [9], while most of the energy efficiency research has been limited to efficient channel sensing approaches only [1]. With wide band spectrum analyzers and the notion of spectrum access systems (that coordinates spectrum assignments) [21] becoming increasing available, soon a DSA end node is liable to have multiple choices for band selection.

In this paper, we show that apart from other factors, band type is an important factor that affects the network energy efficiency given the varying message requirements. First, we propose an architecture for smart connected communities (SCCs) using a small number of DSA enabled devices that form an overlay network on top of the legacy infrastructure. Then, the proposed approach exploits the distinct electro-magnetic (EM) characteristics of different bands in order to intelligently match any message requirement to a suitable band and, hence determines an optimal TTL constrained energy-efficient (TcE) path that achieves the best end-to-end energy efficiency and meets the TTL deadline. We formulate a constrained optimization problem for the determination of TcE path for any given message requirement. We propose a polynomial-time dynamic programming approach for solving the optimization problem where the DSA overlay topology is relatively steady. Compared to the homogeneous band access and spectrum sharing approaches that are restricted to opportunistically access an available channel within a predetermined band, our studies show that the proposed approach using band selection significantly improves the energy efficiency (almost 40\%) while preserving the QoS. Finally, we investigate and discuss some challenges of band selection for a variable overlay topology without global knowledge as a case study. To the best of our knowledge, no prior work has investigated the energy efficient communication through suitable band selection for various message requirements. The benefit of this work is that it provides an easy-to-deploy low cost option to connect sub-urban/rural communities with improved energy efficiency. Moreover, it provides an alternative for improved energy-efficient communication network without further burdening the wired infrastructures or incurring any additional cost, for urban communities.

The rest of the paper is organized as follows. Section 2 reviews the related work. Section 3 presents the proposed architecture for SCCs. Section 4 discusses the distinct EM characteristics of various bands that can be exploited to achieve energy-efficiency. Section 5 presents the proposed band selection approach for a steady DSA topology. Section 6 presents the simulation results, while Section 7 discusses challenges and roadmap for band selection under variable DSA topology, followed by conclusions in Section 8.

\section{RELATED WORK}

We review the related literature for both traditional (non DSA) and DSA/Spectrum sharing approaches for smart connected communities using wireless cyber physical systems. Several standard (nonDSA) wireless communication systems, e.g. DakNet [26], KioskNet [29], have been proposed to provide connectivity by utilizing buses and public service vehicles (equipped with computers with Wi-Fi radio) as mechanical backhaul to provide Internet connectivity to rural or remote communities. In contrast, JaldiMAC [4] utilizes long-range directional WiFi to form a wireless mesh network for providing 
connectivity in sparsely populated areas. Heimerl et al. [13] proposes the deployment of low power GSM based Village Base Station (VBTS) for rural telephony. In [18] and [28], the authors propose the connectivity utilizing hand-held wireless devices for transient conditions. Google [23] introduces the network of balloons traveling on the edge of space, designed to extend Internet connectivity to people in rural and remote areas. However, all these approaches utilize standard wireless technologies such as WiFi, WiMax, GSM base station, VSAT, WLAN, wireless mesh or use a combination of them, to provide connectivity to the rural communities. Since all these devices access a homogeneous band (usually ISM or LTE bands), they suffer from limited coverage, under provisioning, over provisioning and policy constraints as discussed later. Finally, 3GPP/GSM technology providers are also less motivated to invest in expensive wireless spectrum licenses even after government subsidies in developing countries [12].

Recently the researchers have proposed the utilization of TV whitespaces [17], [15], GSM whitespaces [12] for providing rural connectivity. These approaches allow the secondary devices to opportunistically utilize the whitespaces in fixed band, either TV, GSM etc. for data communication. Researchers in [27] proposed cognitive wireless sensor network (CWSN) for energy-efficient sensor network where each sensor harnesses DSA for energy-efficiency. However, CWSN approach for community scale deployments is impractical as it requires every sensor node to be DSA enabled, which is costly. Additionally, such works do not discuss what kind of spectrum is utilized. We show in the paper that restricting the SCC to one such band is not optimally energy-efficient.

\section{PROPOSED ARCHITECTURE}

This section presents key components, network model and communication mechanism for the proposed architecture.

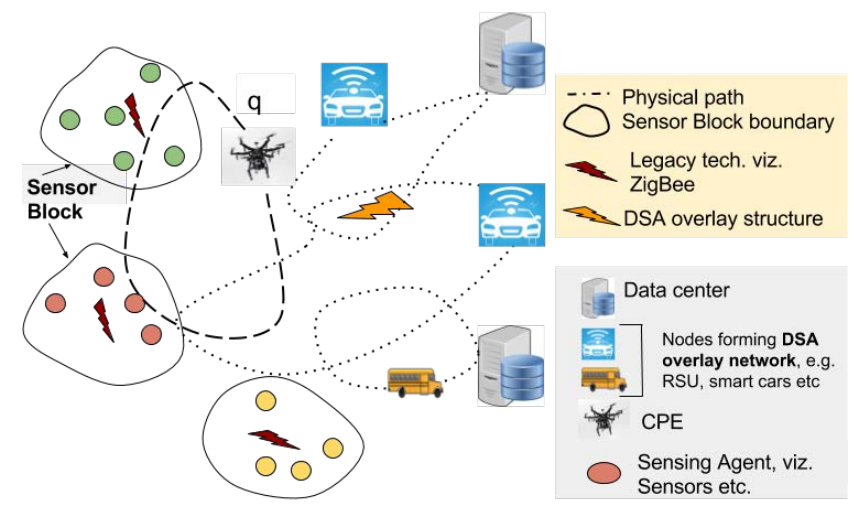

Figure 1: Proposed Architecture

\subsection{Key Components}

Sensor Blocks: A sensor block is a small physical sub network (such as a smart building, smart metering, a crop field etc.) constituted of heterogeneous sensing agents. A sensing agent could be a sensor or a smart device that collects different sensory or contextual data in the form of data, images, audio, video, etc. Such data may have different sizes, ranging from few Kbs (e.g., text, data) to several Mbs (e.g., images, videos) and varying TTL deadlines of few hours (nonreal time). E.g., a rural smart home may constitute temperature, light sensors, smart meters, etc. A smart meter data usually has 1 hour TTL for billing cycle whereas a sensor data on pollution levels, moisture, etc. may have a larger deadline upto $5-6$ hours. Therefore, each sensor block can be a source of various data, each with heterogeneous demands. Owing to the smaller size of the sensor block, it can assumed that the sensing agents within each block form a multi-hop ad-hoc network through low-power short range legacy technologies that forwards its messages to a visiting consumer premise equipment [8]. Unlike previous works [27], our work does not require sensing agents to be DSA enabled making it practical and cost-effective.

Consumer Premise Equipment (CPE): A CPE acts as a proxy between sensor blocks and the DSA overlay network (explained below). A $\mathrm{CPE}$ could be a flying drone or a moving vehicle, that periodically collects various messages from a predetermined set of sensor blocks (denoted by $q \in Q$, where $Q$ is the set of CPEs). This obviates the need of dedicated infrastructure such as separate access points or DSA enabled sensing agents, at each sensor block. We consider that each CPE has two wireless interfaces (i) a low-power short range interface, and (ii) a DSA interface to communicate with the sensor blocks and other DSA enabled devices, respectively.

Data Center A data center is a base station with a back haul link to the final decision making unit, where all the generated messages need to be delivered for the computation and analysis. The data center also has DSA capability.

DSA Overlay Network The DSA overlay network is a collection of

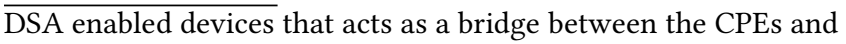
the data center. E.g., fixed road side assistance units (RSU) [31], connected public transportation, smart vehicles, etc. Such a set of DSA enabled devices (denoted by $R$ ) form a wireless DSA overlay network, where each DSA enabled device opportunistically accesses an unoccupied channel on a suitable band for communication. We refrain from discussing the details of the mechanisms used for the discovery and selection of unoccupied (non-interfered) channel within a given band because such issues have been extensively studied in a decade long body of research [2], [6], [9].

We assume that there exists $S$ set of bands where each band $s \in S$ has several (sub) channels. We have considered $S=\{$ TV, LTE, ISM, CBRS , however, it can easily be extended to any other unlicensed bands or licensed bands where DSA is allowed. For example, millimeter bands $(30-300 \mathrm{GHz})$ are being discussed for communication in futuristic 5G technologies [25], [24]. The details of operating frequencies for aforementioned bands are shown in Table 1. Hereafter, a node denotes a DSA enabled device.

While the DSA network topology could be of two types, the focus of this paper is the first one. The types are:

Steady network topology In this case, the overlay nodes are either fixed (i.e., RSUs) or have predictable mobility trajectories (such as public buses, municipal vehicles). Each node shares its trajectory and spectrum availability information with other nodes in the network via a dedicated common control channel or other synchronization techniques as discussed in recent DSA standards [20], [22]. These mechanisms make it possible for each node to possess global 
knowledge about the(i) approximate geographical location and (ii) spectrum availability at every other node.

Variable network topology In this case, DSA overlay nodes may have variable (unpredictable) mobility pattern (e.g. a private vehicle) Hence, a node cannot possess accurate global knowledge of the network. However it may gather the local knowledge about the neighboring nodes via the common control channel.

\subsection{Network Model}

We model the DSA overlay network topology as a directed graph (refer Fig. 3) $G=(V, S, E)$ where $V=Q \cup C \cup R$ is the set of all nodes i.e., CPEs, data center and intermediate nodes, $S$ is the set of band types, and $E \subset(V \times V \times S)$ is the set of all directed links between any two nodes over any common (free) channel in any band type. A link $e_{i j}^{(s)} \in E$ is a directed link from node $i$ to $j$ over band $s \in S$ Hence, there may exist atmost $|S|$ unique links between any given node pair $i, j \in V$. We denote any message traversing through $G$ by $m<u, v, L, T>$ where $u$ is the source, $v$ is the destination, $L$ is the message size and $T$ is the TTL deadline. Now, for a given message $m$, each link is characterized by a tuple $<w_{i j}^{(s)}(L), \hat{t}_{i j}^{(s)}(L)>$, denoting the energy and latency costs, respectively.

\subsection{Communication Mechanism}

As shown in Fig. 2, a CPE $q \in Q$ periodically broadcasts a Hello packet in the predetermined sensor blocks. On receiving the Hello packets, every sensing agent in a sensor block switches from a default energy-saving Sleep (receive only) mode to an Active (transmit and receive) mode, and transmits the messages directly or indirectly via other sensing agents to the visiting CPE $q$. The CPE, then broadcasts Sleep packet in the sensor block such that the sensing agents switch back to energy-saving Sleep mode. Meanwhile, the $\mathrm{CPE}$ selects a suitable energy-efficient band (say, $s \in S$ ). We discuss the band selection approach in sections 5 and 7 for steady and variable DSA overlay topologies, respectively.

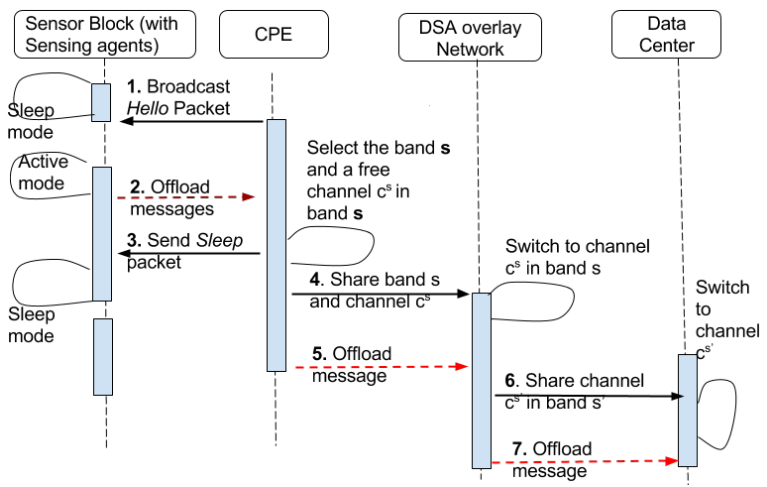

Figure 2: Communication Mechanism

Once the band $s$ is determined at CPE $q$, it shares the chosen band and the channel information (denoted by $c^{(s)}$ ) with any node in the DSA overlay network (or the data center if in range), which then switches to the channel $c^{(s)}$ in chosen band type $s$. Note that a unique node is determined in the steady topology whereas in case of variable topology, $q$ floods the information to every neighboring node and improve the chances of message delivery. After that, the
CPE $q$ transmits the stored messages to the chosen node(s) in the DSA overlay network (or data center if in range) over channel $c^{(s)}$. The similar process goes on nodes until the messages are delivered to the data center.

To meet the main objective of this work (i.e., end-to-end energy efficiency while not sacrificing the QoS), any message $m<$ $u, v, L, T>$ from any node $u$ (e.g, CPE) has to be delivered to the data center $v$ in an energy-efficient manner while guaranteeing the TTL deadline. Therefore, in rest of the paper, we primarily investigate the determination of TTL constrained energy-efficient path (TcE) path $p(u, v)$ through optimal band selection for any given message $m$ such that the end-to-end energy efficiency is maximized and the TTL deadline is met.

\section{TRADE-OFFS BETWEEN BAND SELECTION AND ENERGY EFFICIENCY}

In this section, we first present the energy consumption model for DSA overlay network. Then, we discuss how unique electromagnetic (EM) characteristics offered by various bands can be intelligently exploited to improve the energy efficiency of the DSA overlay network for SCCs.

\subsection{Energy Consumption Model}

For the graph $G$, let a source node $u$ needs to transmit a message $m\langle u, v, L, T\rangle$ to the destination node $v$ which is $d_{u v}$ euclidean distance apart. Suppose, $P_{i}^{(s)}$ is the transmit power at any node $i$ over band $s, \gamma^{(s)}$ is the transmission coverage achieved over a band $s$. Let $\mathbb{R}_{i j}^{(s)}$ be effective bit rate achieved from any node $i$ to $j$ over band $s$. Please note that different bands have different EM characteristics that may result into different allowable transmit power, coverage and effective bit rates (explained later in this section).

The DSA overlay network is influenced by various EM factors such as operational frequency, bandwidth, path loss factor, interference etc. Therefore, the energy consumption model must consider all these factors. Now, given a path $p(u, v)$ for the message $m$, the end-to-end energy consumption is given by:

$$
w_{u v}(L)=\sum_{e_{i j}^{(s)} \in p(u, v)} w_{i j}^{s}(L)=\sum_{e_{i j}^{(s)} \in p(u, v)} P_{i}^{(s)} \times t_{i j}^{(s)}(L)
$$

where $e_{i j}^{(s)}$ denotes an intermediate edge in path $p(u, v)$. The message transmission time $\left(t_{i j}^{(s)}(L)=\frac{L}{\mathbb{R}_{i j}^{(s)}}\right)$, defined as the time taken to deliver the entire message $m$ from node $i$ to node $j$ over a band $s$ i.e., edge $e_{i j}^{(s)}$ (explained later in section 4.3). Now, considering that every node is restricted to utilize the same band, termed as homogeneous band access then, $w_{u v}(L)$ is given by

$$
w_{u v}(L)=P_{u}^{(s)} \times t_{i j}^{(s)}(L) \times H
$$

$H$ is the number of hops required to transfer the message from node $u$ to node $v$, calculated as $H=\frac{d_{u v}}{\gamma^{(s)}}$.

\subsection{Operating Frequency and Energy Efficiency}

We know that the received signal strength at a receiver should meet some reception threshold $\tau$ to decode a signal accurately. The 
generalized Frii's transmission equation provides the relationship between the received power at receiver $j$ and transmit power from transmitter $i$ over any channel with a representative frequency ${ }^{1}$ $f^{(s)}$ in band $s$,

$$
P_{j}^{(s)}=P_{i}^{(s)} G_{i} G_{j}\left(\frac{\mathbb{C}}{4 \pi f^{(s)} d}\right)^{\alpha} \geq \tau
$$

where, $G_{i}$ and $G_{j}$ are the transmitter and receiver antenna gains, $\alpha$ is path loss exponent, $\mathbb{C}$ is the speed of light, $d$ is the distance between $i$ and $j, P_{i}^{(s)}$ is the transmit power of $i, P_{j}^{(s)}$ is the received power at $j$. Now let a constant $\phi=G_{i} G_{j}\left(\frac{\mathbb{C}}{4 \pi}\right)^{\alpha}$.

Then, from $P_{j}^{(s)}=\phi \frac{P_{i}^{(s)}}{\left(f^{(s)} d\right)^{\alpha}}$, it is evident that for higher frequencies, the transmit power $P_{i}^{(s)}$ ought to be increased to maintain the same $P_{j}^{(s)} \geq \tau$, even though the distance $d$ is unchanged. The resultant increased transmit power causes more energy consumption (refer. Eqs. 1 and 2). Alternatively, if transmit power is not increased, then $\tau$ is only met at a lower distance. Hence, more intermediate hops would be required to traverse the same distance $d$, leading to the additional transmission and reception operations at each intermediate hop which causes higher energy expenditure.

Moreover, the lower frequencies have larger wavelengths yielding better obstacle and wall penetration capabilities. Hence, the lower frequencies are less error prone, thus reducing (re) transmission overheads and enabling better non line-of-sight connectivity. Among the bands that allow DSA, TV and LTE bands have lower frequency allocations than traditionally used unlicensed band (2.4 $\mathrm{GHz}$ ). Hence, these bands should be more energy efficient.

However, lower frequencies come at a trade-off in the sense that they usually have much lesser bandwidth of $6 \mathrm{MHz}$ as opposed to LTE (upto $20 \mathrm{MHz}$ ), and CBRS (upto $40 \mathrm{MHz}$ ). Hence for larger message sizes, the message transmission time (refer Eq. 6) will be higher. Supporting high data rate applications are also challenging for lower frequencies offering smaller bandwidths. However, frequencies offering higher bandwidths will reduce the transmission time for a given message size and save energy. An important research question, therefore is that whether the reduction in the transmission time for higher frequencies offsets the gain in energy efficiency due to reduced hop count.

\subsection{Bandwidth and Energy Efficiency}

The relationship of bandwidth with the dissipated power could be understood through Rayleigh-Parseval Equation:

$$
P_{\text {dis }}^{(s)}=\int_{f_{1}^{(s)}}^{f_{2}^{(s)}} \mathbb{S}_{i}\left(f^{(s)}\right)
$$

where $\mathbb{S}_{i}$ is the power spectral density, $\Delta f^{(s)}=\left(f_{2}^{(s)}-f_{1}^{(s)}\right)$ is the bandwidth of any channel in the band type $s$ and $P_{r e q}^{(s)}$ is the total power dissipated over band $s$. From Eq. 4 , it is clear that a channel offering higher bandwidth causes larger power dissipation. Given a fixed transmission time for any message, higher bandwidth channels will consume more energy than lower ones. The typical bandwidths for various spectrum is listed in Table 1 .

\footnotetext{
${ }^{1}$ For example, $f_{s}=2.4 \mathrm{GHz}$ for the frequencies in the range $2.412-2.462 \mathrm{GHz}$ used by legacy WiFi devices in ISM Band
}

Table 1: Spectrum profile

\begin{tabular}{|c|c|}
\hline Spectrum & Bandwidth \\
\hline $54-216 \mathrm{MHz}$ (VHF TV Band) & $6 \mathrm{MHz}$ \\
\hline $470-698 \mathrm{MHz}$ (UHF TV Band) & $6 \mathrm{MHz}$ \\
\hline $698-806 \mathrm{MHz}$ (700 MHz Band/FirstNet) & $6 \mathrm{MHz}$ \\
\hline $700-800 \mathrm{MHz} / 1700-2100 \mathrm{MHz}$ (LTE Band) & $5-20 \mathrm{MHz}$ \\
\hline $902-928 \mathrm{MHz}$ (ISM Band) & $3-8 \mathrm{MHz}$ \\
\hline $2.4-2.5 \mathrm{GHz}$ (ISM Band) & $2-50 \mathrm{MHz}$ \\
\hline $3.5-3.7 \mathrm{GHz}$ (CBRS Band) & $10-40 \mathrm{MHz}$ \\
\hline $5.7-5.8 \mathrm{GHz}$ (ISM Band) & $20-80 \mathrm{MHz}$ \\
\hline
\end{tabular}

On contrary, from Shannon-Hartley theorem, the effective bit rate $\left(\mathbb{R}_{i j}^{(s)}\right)$ between transmitter $i$ and receiver $j$ over any channel in band $s$ with representative freq. $f^{(s)}$ is given by

$$
\mathbb{R}_{i j}^{(s)}=B^{(s)} \log _{2}\left(1+\chi_{i j}^{(s)}\right)=B^{(s)} \log _{2}\left(1+\phi \frac{P_{i}^{(s)}}{\left(f^{(s)} d\right)^{\alpha} N^{(s)}}\right)
$$

where $B^{(s)}$ is the bandwidth of any channel in band type $s$ and $\chi_{i j}^{(s)}=P_{j}^{(s)} / N^{(s)}$ is the ratio of communication signal power to the interference and noise at the receiver (SINR). Rest of the notations are the same as above.

For a given fixed message with $L$ bits, the total transmission time is calculated as follows:

$$
t_{i j}^{(s)}(L)=\frac{L}{\mathbb{R}_{i j}^{(s)}}=\frac{L}{B^{(s)} \log _{2}\left(1+\phi \frac{P_{i}^{(s)}}{\left(f^{(s)} d\right)^{\alpha} N^{(s)}}\right)}
$$

From Eq. 5, given that the SINR is unchanged, a higher bandwidth channel would offer a higher effective bit rate, thereby decreasing the required transmission time.

Hence, from the relationship between bandwidth and req. power (see Eq. 4) and effective bit rate (see Eq. 5), the higher the channel bandwidth is, the higher is the dissipated power but the lower is the transmission time. Thus, the trade-off is whether to choose a high bandwidth channel that reduces the transmission time and increases the dissipated power or vice-versa for any given message.

\subsection{Interference and Energy Efficiency}

Increased cross device interference will increase the noise floor as more and more devices coexist in the unlicensed spectrum (2.4 $\mathrm{GHz}$ ) and GSM/LTE (1700-2100 MHz) band. Therefore, from Eq. 5, it is evident that SINR will decrease. To maintain a certain required SINR, the transmit power needs to be increased. However, switching to any other band type using DSA would be able to maintain the same SINR with a low transmit power, thus saving energy.

\subsection{Packet Size and Energy Efficiency}

Packet sizes in existing low-power short range technologies $(2.4$ $\mathrm{GHz}$ ) are usually small (maximum size 128 bytes) because the bit error rate is very high. However, communication through lower frequency bands experiences less bit error rate (as explained in Section 4.2). Hence, larger packet sizes can be employed which would reduce per-packet overheads such as headers and preambles, as shown in [16]. Moreover, the higher packet size would decrease the overall message transmission time. Both these factors promises to improve the energy-efficiency. 


\section{BAND SELECTION WITH GLOBAL KNOWLEDGE}

In this section, we discuss the proposed band selection approach for steady network topology where each node has the global knowledge of the network. The main idea is to intelligently match the message $m<u, v, L, T>$ to a suitable band at each intermediate node between the source $u$ and destination $v$ so as to construct the TTL constrained energy-efficient (TcE) path $p(u, v)$. We first present two lemmas to show that the greedy approaches that locally optimizes the band selection may not always guarantee end-to-end energy efficiency for any message $m$ (i.e., optimal TcE path $p(u, v)$ ).

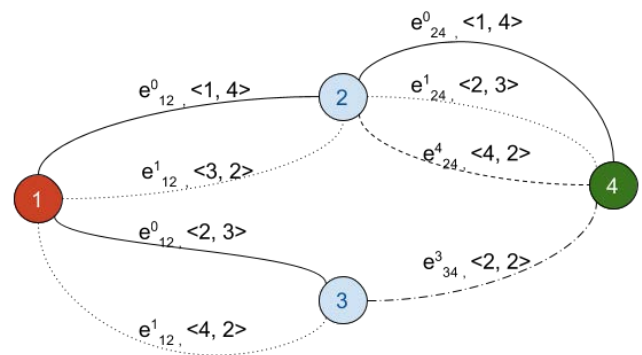

Figure 3: Network Model. $e_{12}^{0}$ and $e_{12}^{1}$ denote links between node 1 and 2 over band type 0 and 1 , resp. The tuples $<1,4>$ and $<3,2>$ signify energy and latency costs across links $e_{12}^{0}$ and $e_{12}^{1}$, resp.

Lemma 1: Any path $p^{\prime}(u, v)$, which is constructed by selecting locally optimal band at any intermediate node, may not always yield the optimal TcE path $p(u, v)$ for any given message $m\langle u, v, L, T\rangle$.

Proof: Let us consider the network (Fig. 3) where node 1 and node 4 are the source and destination nodes, respectively, and a message $m$ with $T=5$, initially at node 1 . Then, the path $p^{\prime}(1,4)$ constructed by choosing locally optimal band at each intermediate node would be

$$
1 \stackrel{e_{12}^{0},<1,4>}{\underset{\text { Band } 0}{\longrightarrow}} 2 \nrightarrow 4
$$

There exists no solution i.e., optimal TcE path $p^{\prime}(1,4)$ that meets the TTL deadline $T=5$. Here, the optimal TcE path $p(1,4)$ is as follows:

$$
1 \stackrel{e_{12}^{0},<2,3>}{\underset{\text { Band } 0}{\longrightarrow}} 3 \stackrel{e_{34}^{3},<2,2>}{\text { Band } 3} 4
$$

Hence, the greedy approach of selecting optimal band at each intermediate node locally, do not always guarantee the construction of optimal TcE path for any message $m$.

Lemma 2: Any path $p^{\prime}(u, v)$, which is constructed by selecting the best unique (homogeneous) band at each intermediate node may not always provide the optimal TcE path $p(u, v)$ for any message $m<u, v, L, T>$.

Proof: It can be proved easily as before (from above example network as shown in Fig. 3).

We present these lemmas to show that the construction of optimal TcE path for any given message is not straightforward. Neither of aforestated locally optimized approaches always guarantee the construction of optimal TcE path. Hence, we formulate the following optimization problem and propose a dynamic programming approach to find the optimal solution in polynomial time.

\subsection{Problem Formulation}

This section formulates a constrained optimization problem where the objective is to determine the TTL constrained energy-efficient (TcE) path for any message $m<u, v, L, T>$.

Note that although we solve the optimization problem for a given message $m$, it will also work well for concurrent messages. This is because a band is composed of several channels, that can be simultaneously accessed by concurrent messages (should they end up choosing the same band in the same geographical region); unless in the unlikely rare event that the number of such messages exceed the total number of available channels. Moreover, there may be a small channel access delay due to channel bargaining within a selected band, which is negligible in the range of micro seconds, therefore the optimization problem ignores it.

The determination of an energy-efficient path for a message $m<u, v, L, T>$ can now be formally defined: Given a directed graph $G=(V, S, E)$, an energy cost $w_{i j}^{(s)}(L)$ and a latency cost $\hat{t}_{i j}^{(s)}(L)$ to transmit a message $m<u, v, L, T>$ along a link $e_{i j}^{(s)} \in E$, a source node $u \in V$, a destination $v \in V$, the optimization problem is:

$$
\min _{p(u, v) \in P^{\prime}(u, v)} \sum_{e_{i j}^{(s)} \in p(u, v)} w_{i, j}^{(s)}(L)
$$

where $P^{\prime}(u, v) \subset P(u, v)$ is the set of all paths from the source node $u$ to the destination node $v$ which meets the constraints:

(i) SINR constraint For the successful decoding of any message $m$ at $j$, the achieved SINR $\chi_{i j}^{(s)}$ at receiver $j$ must be greater than or equal to prespecified SINR threshold $\chi_{t h}$.

$$
\chi_{i j}^{(s)} \geq \chi_{t h}
$$

(ii) Power constraint As per the FCC guidelines, the maximum transmit power at any transmitter $i$ must be less than or equal to the maximum EIRP $P_{\text {max }}^{(s)}$ over any band $s \in S$.

$$
P_{i}^{(s)} \leq P_{\max }^{(s)}
$$

(iii) Transmission coverage constraint For the successful data communication over any intermediate link $e_{i j}^{(s)} \in p$, the euclidean distance $d_{i j}$ must be less than or equal to the transmission coverage $\gamma^{(s)}$ achieved in the chosen band $s$.

$$
\gamma^{(s)} \geq d_{i j}
$$

(iv) Unique spectrum constraint Any transmitter-receiver node pair $i, j \in V$ must tune to a common available channel in a unique band $s \in S$.

$$
\sum_{s \in S} e_{i j}^{(s)} \leq 1, \quad \forall e_{i j}^{(s)} \in p
$$

(v) TTL constraint Any message must be delivered within its TTL deadline from its source node $u$ to destination $v$. Hence, the total latency cost $\left(\sum_{e_{i j}^{(s)} \in p(u, v)} \hat{t}_{i j}^{(s)}(L)\right)$ for the path i.e., sum of message 
transmission delay $\left(t_{i j}^{(s)}=L / \mathbb{R}_{i j}^{s}\right)$, propagation delay $\left(d_{i j} / \mathbb{C}\right)$, and queuing delay $(\epsilon)$, must be less than or equal to $T$.

$$
\sum_{e_{i j}^{(s)} \in p(u, v)} \frac{L}{\mathbb{R}_{i j}^{(s)}}+\frac{d_{i j}}{\mathbb{C}}+\epsilon \leq T
$$

where $\mathbb{C}$ is the propagation speed of any spectrum (usually speed of the light). Note that the propagation and queuing delays are usually negligible compared to the message transmission delay.

\subsection{Proposed TcE Algorithm}

We propose a dynamic programming (DP) approach for the above constrained optimization problem. The proposed algorithm intelligently matches the message $m$ to an optimal (energy-efficient) band such that the total energy cost incurred in path $p(u, v)$ is minimized whereas the TTL and other constraints are met. The proposed TcE algorithm is inspired from a well-known Floyd-Warshall algorithm for all pair shortest paths.

Now, for a message $m$ with $L$ bits and TTL $T$ at each source node $i \in V$ over band $s \in S$, the graph $G(V, S, E)$ can be represented by a 3D adjacency matrix $A:|V| \times|V| \times|S|$ defined as follows:

$$
A[i, j, s]=\left\{\begin{array}{lc}
<0,0>, & i=j \\
<w_{i j}^{(s)}(L), t_{i j}^{(s)}(L)>, & i \neq j, \quad \text { and constraints } \\
& \text { in Eqs. 8, 9, 10, and 11 are met } \\
<\infty, \infty> & \text { otherwise }
\end{array}\right.
$$

An element in $A[i, j, s]$ is an ordered pair $<., .>$ where the first and second parts denote the energy and latency costs, respectively for a message $m$ along the link $e_{i j}^{s}$ from node $i$ to $j$ over band $s$. The $\mathrm{TcE}$ algorithm is based on the notion of intermediate node, which is defined as follows:

Definition 1 (Intermediate node) Given a path $p=<1,2, \ldots,(|l|-$ 1), $|l|>$, an intermediate node is any node $i$ such that $i=2, \ldots,(|l|-$ 1), i.e., any node except 1 and $|l|$.

The TcE algorithm is based on the following observation. Let us consider a subset of nodes $\{1, \ldots k\}$. For any node pair $i, j \in V$, let $p$ be the path with least energy cost, out of all paths between $i$ and $j$ whose intermediate nodes are in $\{1, \ldots k\}$. Then, there are two possible cases.

$k$ is not an intermediate node of $p$, then, all the intermediate nodes of path $p$ are in $\{1, \ldots, k-1\}$. Hence, the energy-efficient path from $i$ to $j$ with intermediate nodes $\{1, \ldots, k\}$ is also the energyefficient path from $i$ to $j$ with intermediate nodes in $\{1, \ldots, k-1\}$

$k$ is an intermediate node of $p$, then, $p$ can be broken into two sub-paths: one sub-path $p_{1}$ from $i$ to $k$ and another sub-path $p_{2}$ from $k$ to $j$. Since, $p$ is the energy-efficient path, $k$ can only appear once in $p$. Therefore, $k$ can not be an intermediate node of $p_{1}$ or $p_{2}$. Moreover, by optimality principle (that states that the sub-paths of shortest path are also the shortest paths), both $p_{1}$ and $p_{2}$ are the shortest i.e., energy-efficient paths between $i$ and $k$, and $k$ and $j$, respectively, with intermediate nodes in the set $\{1, \ldots k-1\}$.

We define the sub problems used by the DP approach as follows. Let $w_{i j}^{(k, T)}$ be the energy cost of the energy-efficient path from node $i$ to $j$ that meets TTL $T$ and all the intermediate nodes are in the node set $\{1,2, \ldots, k\}$. Now, we utilize above observations to define the recursive relation between the solutions of sub-problems, and ultimately lead to the solution of the overall problem.

Base case:

1. $k=0$, There are no intermediate nodes in the path, hence for each $i, j \in V$,

$$
w_{i j}^{(0, T)}=\left\{\begin{array}{l}
\min _{s \in S} A[i, j, s] \cdot w_{i j}^{(s)}(L), \text { if } \exists s \in S \text { s.t } \hat{t}_{i j}^{(s)} \leq T \\
\infty \quad \text { otherwise }
\end{array}\right.
$$

2. $T=0$, there is no path, hence $w_{i j}^{(k, 0)}=\infty$, for each $i, j \in V$.

Inductive case, $k>0$ and $T>0$, we can select a path with the least energy cost either using $k$ as intermediate node or not, however meets the TTL deadline $T$.

$$
w_{i j}^{(k, T)}=\min _{t=1 \ldots T}\left(w_{i j}^{(k-1), t}, \min _{t_{1}=0 \ldots t}\left(w_{i k}^{(k-1), t_{1}}+w_{k j}^{(k-1),\left(t-t_{1}\right)}\right)\right)
$$

When $k=|V|$ and $t=T$, then we have the final solution, which is the total energy cost for energy-efficient path for each sourcedestination pair with TTL $T$ i.e., $W^{(|V|, T)}=\left(w_{i j}^{(|V|, T)}\right), \forall i, j \in V$.

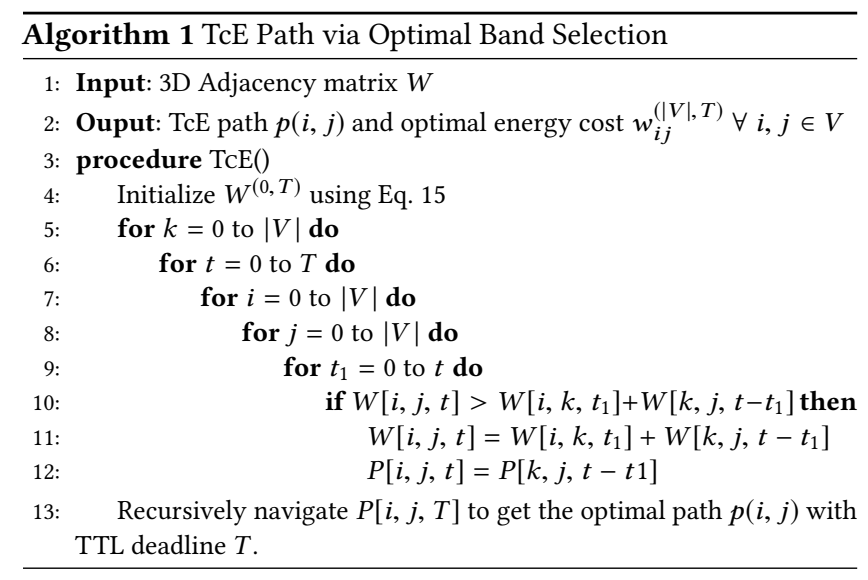

Algorithm Description: The TcE algorithm uses a series of 3D matrices $W^{(k, t)}$ for $k=0, \ldots|V|$ and $t=0, \ldots T . W^{(k, t)}$ contains the elements $w_{i j}^{(k, t)}$, that is the TTL $(1 \leq t \leq T)$ constrained energyefficient path between $i$ and $j$ using the intermediate nodes in $\{1, \ldots k\}$. Also, we keep track of the optimal TcE path in another matrix $P_{i j}^{(k, t)}$. The algorithm has following steps:

Step 1 (Initialization): The algorithm initializes matrix $W^{(0, T)}$ to $A$ under following conditions:

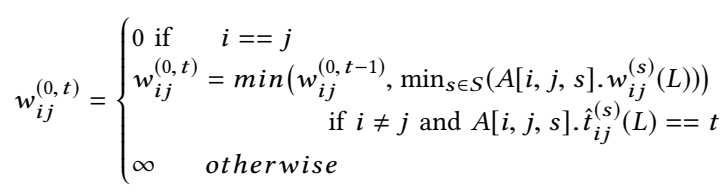

Step 2 (TcE path via band selection): After the initialization, the algorithm applies the recursive formula to calculate $W^{(k, t)}$ given $W^{\left(k-1, t_{1}\right)}$ and $W^{\left(k-1, t-t_{1}\right)}$, as shown in Algorithm 1. Also, the path tracking matrix $P$ is updated recursively (Lines 5 - 12). 
Finally, any source node $i$ delivers the message to the destination node $j$ via the computed optimal TcE path $p(i, j)$ (obtained from path tracking matrix $P$ ). The algorithm time complexity is $O\left(|V|^{3} \times T^{2}\right)$ where $|V|$ is the number of nodes, and $T$ is the TTL deadline.

\subsection{Discussion on Cost Benefit Analysis}

Many detractors of sustainability argue that green computing incurs higher capital expenditure and operational cost which drains the economy/revenue and makes such designs infeasible in practice. However, we believe that our proposed approach of opportunistic band selection to realize SCCs, is free from most such disadvantages due to following reasons: (i) The use of DSA enabled access philosophy does not require a provider of SCC to own dedicated spectrum licenses, worth millions of dollars regardless variable traffic volume., (ii) Multiple smart communities can be connected with a small set of DSA overlay nodes where each DSA nodes' average cost is around $\$ 500-\$ 700$. In contrast, our design obviates the need for new dedicated infrastructure such as cellular base station ( $\$ 200,000 /$ station) or LTE stations ( $\$ 100,000 / 3$ sectors) in rural areas, and the need for extensive wire line communications to increase the available bandwidth in urban areas. Also burdening of existing networks whether wired or wireless is obviated, and (iii) Some real examples of micro-telco models of DSA based deployment has already taken place in relatively rural areas in the developing world such as Papua [12], Mexico [10], and Philippines [14].

To summarize, there is sufficient practical evidence to suggest that the equipment and operational costs are far less compared to that of traditional approaches. The major hurdles are the policy issues which surround spectrum planning. However, significant policy breakthroughs [20], [22] in spectrum policy has been made in recent years and DSA devices mounted over drones, public transport [7], and private smart cars [35] are going to be a reality.

\section{SIMULATION RESULTS}

In this section, we evaluate the proposed band selection approach against homogeneous band access approaches, for steady DSA overlay topology in terms of following performance metrics: (i) Energy Efficiency - the average amount of energy consumed to transfer a generated message from a sensor block to the intended data center, (ii) Message Delivery Ratio (MDR) - the fraction of total messages delivered to the data center within the TTL deadline, to the total generated messages at all sensor blocks, and (iii) Network Latency - the average delay incurred (i.e., total transmission delay, propagation delay and queuing delay at each intermediate node) in delivering all messages, each from its block to the data center.

Each of the aforestated performance metric is evaluated against the following parameters: (i) varying message size, (ii) varying TTL deadline, and (iii) varying source-destination pair distance. The first two parameters represent the heterogeneity in various messages, while the third one represents the heterogeneity in geographical distance between the sensor blocks and data centers.

Simulation Settings: We develop a Java simulator to simulate a SCC scenario with 5 data centers, 15 CPEs, 30 sensor blocks, each with $50-100$ sensing agents. On average, each CPE periodically visits $2 \mathrm{CPEs}$ with a round trip time of 1 hour. The steady DSA overlay topology consists of 200 DSA nodes (e.g., public buses, RSUs etc.). $50 \%$ of overlay nodes (imitating RSUs) are randomly placed and are static whereas other overlay nodes (imitating public buses) move over a fixed trajectory. We consider that each DSA enabled node has the knowledge of location and spectrum availability at every other nodes in the network. Unless otherwise stated, the message size, TTL deadline and farthest distance between sensor blocks and data centers are taken as $250 \mathrm{MB}, 6$ hours, and $45 \mathrm{Km}$ for the experiments, respectively.

Each band has a FCC mandated maximum allowable transmit power which can not be exceeded by any node operating on a DSA basis. Hence, we consider a transmit power $(1 \mathrm{~W}(30 \mathrm{dBm}))$ which is allowable on a secondary basis, in all ISM, TV, LTE and CBRS bands. The values of other controlling parameters are as follows: path loss factor $\alpha=3.5$ (sub-urban area), $\operatorname{SINR}=-75 \mathrm{dBm}\left(3.16 \times 10^{-8} \mathrm{~mW}\right.$ ), and received power threshold $(\tau)=-20 \mathrm{dBm}(0.01 \mathrm{~mW})$.

\subsection{Energy Efficiency}

Figs. 4(a), 4(b), and 4(c) clearly show that the energy consumed by our proposed approach is significantly lower than all homogeneous band approaches across various message sizes, TTL deadlines and source-destination distances. The average amount of energy saved by our approach is $36.2 \%, 47 \%$, and $42.2 \%$ against all other approaches combined (whereas 15\%,20\% and 13\% against the best one), for various message sizes, TTL deadlines and distances, respectively. This is because our approach chooses a suitable band at each intermediate node for enhancing energy efficiency unlike scenarios where the node is restricted to access only one predetermined band.

Note that in Fig. 4(c), the energy consumed is $0 \mathrm{~J}$ for distance $75 \mathrm{Km}$ for ISM and CBRS bands. This is because there exist no intermediate nodes in the communication range, that could forward the message from the sensor block towards the data center. Hence, no energy was consumed at any node in the DSA overlay network. However, the MDR is negligible $(\approx 0 \%)$ (refer. Fig. 5(c)), hence defeating the purpose of SCC. Besides this, the proposed approach always achieves the highest energy efficiency, and meets the QoS.

\subsection{Message Delivery Ratio}

As illustrated in Figs. 5(a), 5(b), and 5(c), the proposed approach yields either comparable or better message delivery ratio (MDR) compared to that of homogeneous band approaches, irrespective of varying message sizes, TTL deadlines and source-destination pair distances, respectively. The improvement in MDR for our approach is almost $15 \%$ for message size ( $>500 \mathrm{Mb}), 17 \%$ for TTL deadline $(<3$ hours) and $10 \%$ for distance $(>50 \mathrm{Km})$, compared to the best of homogeneous bands, i.e., LTE Band for first two cases and TV Band for the third one. This is because the proposed TcE algorithm simultaneously accounts for TTL besides enhancing energy savings, and thereby always guarantees the highest MDR for any heterogeneous message. However, the homogeneous band approach do not have the flexibility of choosing any other band than the pre-specified one, thereby incurring poor MDR.

\subsection{Network Latency}

Figs. 6(a), 6(b), and 6(c) show that the proposed approach achieves better network latency compared to rest of the approaches, irrespective of message and geographical heterogeneity. This is because our 


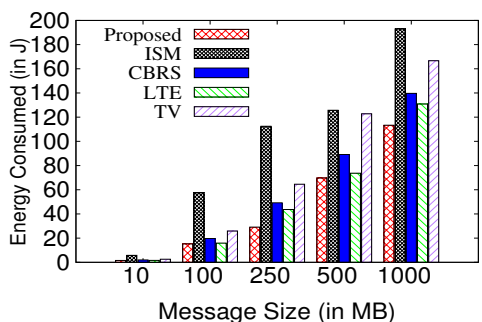

(a)

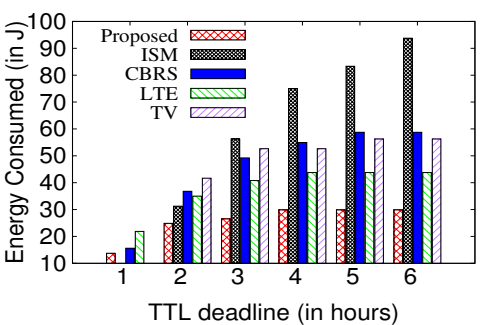

(b)

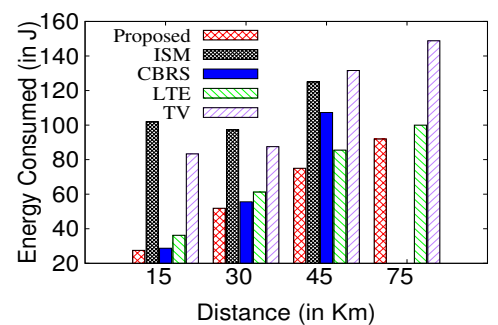

(c)

Figure 4: Energy Efficiency: (a) Message Size, (b) TTL, and (c) Dist. between Sensor block and data center

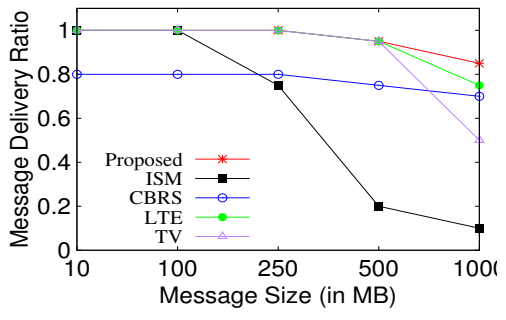

(a)

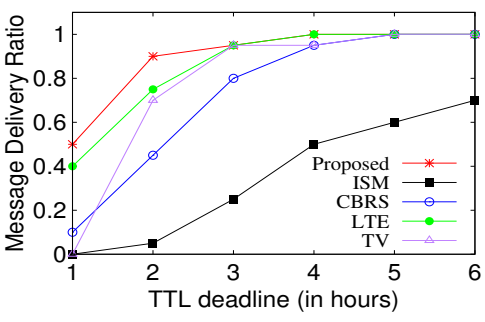

(b)

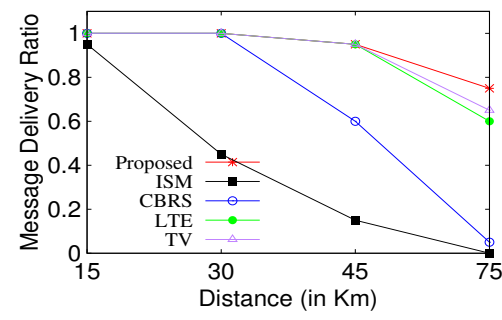

(c)

Figure 5: Message Del. Ratio: (a) Message Size, (b) TTL, and (c) Dist. bet. Sensor block and data center

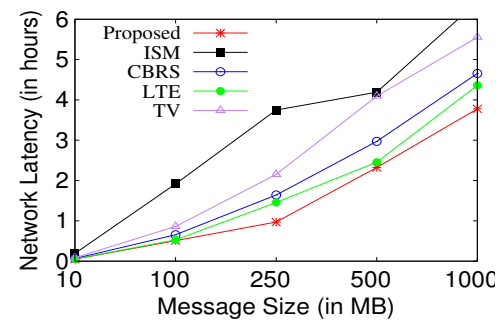

(a)

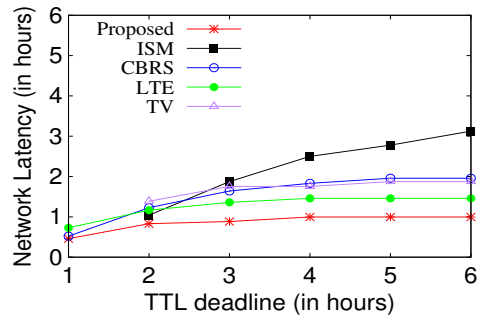

(b)

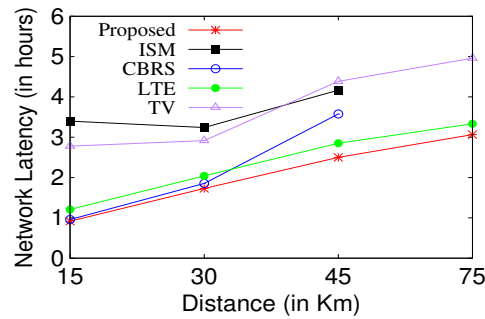

(c)

Figure 6: Network Latency: (a) Message Size, (b) TTL, and (c) Dist. between Sensor block and data center

approach attempts to choose the path with least message transmission time while determining the TcE path (from Eq. 2). Specifically, our approach improves the network latency by almost $16 \%, 23 \%$ and $14 \%$ compared to the best of homogeneous band approaches (i.e. LTE), for varying message sizes, TTL deadlines and geographical distances, respectively. Note that the network latency for ISM and CBRS bands are not shown for distance $=75 \mathrm{Km}$ (refer. Fig. 6(c)). This is because their network latency is infinity as no message has been successfully delivered within its TTL deadline (see Fig. 5(c)).

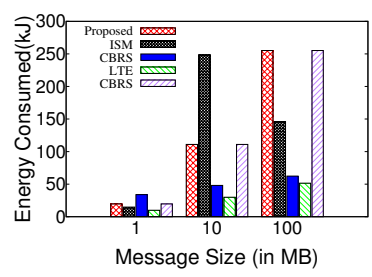

(a)

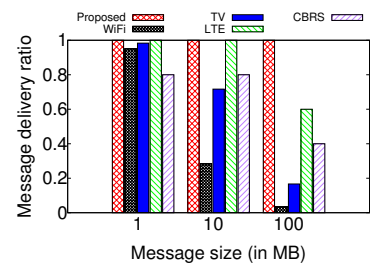

(b)
Figure 7: Variable DSA Network: a. Energy Efficiency, and b. Message Delivery Ratio

\section{VARIABLE DSA TOPOLOGY: A CASE STUDY}

To realize the true power of ubiquitous connectivity, we believe private smart vehicles could also be harnessed as members of the DSA overlay network. However, since mobility in such cases can not be predicted, constructing a global knowledge may be impossible. We investigate certain challenges and a road map for energy efficient band selection under such a variable topology. Due to lack of global knowledge, we investigate a simple greedy approach that attempts to choose the locally optimized energy-efficient band at any given node for any message, hopefully leading to the end-to-end energy efficiency while meeting the TTL deadline.

The greedy algorithm chooses a certain band $s$ at any given node $u$ for any message $m$ only if following conditions are met: (i) if the next hop is the destination, or (ii) the energy consumed over band $s$ is the least, the latency cost is less than the TTL and there exists at least two nodes in the communication range over spectrum $s$. These conditions are important to improve the chances of successful data delivery to the destination node. Once the suitable band is chosen at the current node, it sends a message copy over chosen band $s$ to all neighboring nodes that lie in the communication range. The 
time complexity of the algorithm is $O(|V| \times|S|)$ where $V$ and $S$ are the set of nodes and band types, respectively.

Challenges and Future Roadmap Now we investigate the performance of the greedy local optimization in variable DSA topology, and draw key challenges and a future roadmap for further research. From Fig. 7(b), it is evident that though the proposed greedy approach outperforms others in terms of MDR, it suffers heavily in terms of energy efficiency compared to that of other homogeneous band access approaches (refer Fig. 7(a)). This is mainly due to various contextual factors such as unpredictable node mobility patterns, node contact behaviors etc. The results are similar for other two parameters (Plots not shown here).

We conclude that a locally optimized greedy solution alone is not an apt solution to improve end-to-end energy efficiency via dynamic band selection. In fact, the optimization under that case should be complemented by an efficient Delay Tolerant Routing protocol that reduces the unpredictability of determining the best set of intermediate nodes by learning geographical contextual factors and contact mobility patterns. Therefore, we plan to investigate the contextual factors and come up with a spectrum and mobility aware approach that improves the end-to-end energy efficiency for any given message that also meets the TTL deadline in such a challenging variable DSA topology.

\section{CONCLUSION}

In this paper, we proposed a novel architecture that uses a small number of DSA enabled devices for forming a DSA overlay network on top of the legacy infrastructure; to enhance end-to-end energy efficiency while guaranteeing the QoS. We also discussed how EM characteristics can be exploited for intelligent matching of any message requirement to a suitable band. Moreover, we formulated a constrained optimization problem and proposed a dynamic programming approach for determining TTL constrained energyefficient (TcE) path for any given message with heterogeneous requirement. Compared to homogeneous band access approaches, the proposed approach excels in terms of energy efficiency while guaranteeing the QoS, irrespective of message and geographical heterogeneity, thus bypassing under and over-provisioning issues.

\section{ACKNOWLEDGMENTS}

This research is partially supported by the NSF grants CNS-1545037, CNS-1545050, and NATO grant G4936. We would like to acknowledge Rishab Singhal for his assistance with the experiments.

\section{REFERENCES}

[1] S. Agarwal and S. De. 2016. eDSA: Energy-efficient dynamic spectrum access protocols for cognitive radio networks. IEEE Transactions on Mobile Computing 15, 12 (2016), 3057-3071.

[2] I. F Akyildiz, W. Y Lee, M. C Vuran, and S. Mohanty. 2006. NeXt generation/dynamic spectrum access/cognitive radio wireless networks: A survey. Computer networks 50, 13 (2006), 2127-2159.

[3] J. Bae, E. Beigman, R. Berry, M. L. Honig, H. Shen, R. Vohra, and H. Zhou. 2008 Spectrum markets for wireless services. In 3rd IEEE Symposium on New Frontiers in Dynamic Spectrum Access Networks (DySPAN). 1-10.

[4] Y. Ben-David, M. Vallentin, S. Fowler, and E. Brewer. 2010. JaldiMAC: taking the distance further. In Proceedings of the 4th ACM workshop on networked systems for developing regions (NSDR). 2:1-2:6.

[5] F. Bonomi, R. Milito, P. Natarajan, and J. Zhu. 2014. Fog computing: A platform for internet of things and analytics. In Big Data and Internet of Things: A Roadmap for Smart Environments. Springer, 169-186.
[6] S. Brahma and M. Chatterjee. 2012. Spectrum sharing in secondary networks: A bargain theoretic approach. In IEEE Wireless Communications and Networking Conference (WCNC). 1331-1336.

[7] L. Cheng, B. E. Henty, D. D. Stancil, F. Bai, and P. Mudalige. 2007. Mobile vehicleto-vehicle narrow-band channel measurement and characterization of the 5.9 $\mathrm{GHz}$ dedicated short range communication (DSRC) frequency band. IEEE fournal on Selected Areas in Communications 25, 8 (2007).

[8] S. Debroy, S. Bhattacharjee, and M. Chatterjee. 2015. Spectrum map and its application in resource management in cognitive radio networks. IEEE Transactions on Cognitive Communications and Networking 1, 4 (2015), 406-419.

[9] S. Debroy, S. De, and M. Chatterjee. 2014. Contention based multichannel MAC protocol for distributed cognitive radio networks. IEEE Transactions on Mobile Computing 13, 12 (2014), 2749-2762.

[10] H. Galperin and F. Bar. 2006. The microtelco opportunity: evidence from Latin America. Information Technologies \& International Development 3, 2 (2006), 73-86.

[11] M. Ghamari, B. Janko, R. S. Sherratt, W. Harwin, R. Piechockic, and C. Soltanpur. 2016. A survey on wireless body area networks for ehealthcare systems in residential environments. Sensors 16, 6 (2016), 831

[12] S. Hasan, K. Heimerl, K. Harrison, K. Ali, S. Roberts, A. Sahai, and E. Brewer. 2014. GSM whitespaces: An opportunity for rural cellular service. In IEEE International Symposium on Dynamic Spectrum Access Networks (DYSPAN). 271-282.

[13] K. Heimerl and E. Brewer. 2010. The village base station. In Proceedings of the 4th ACM Workshop on Networked Systems for Developing Regions. 14.

[14] K. Heimerl, S. Hasan, K. Ali, E. Brewer, and T. Parikh. 2013. Local, sustainable, small-scale cellular networks. In Proceedings of the 6th ACM Int'l Conf. on Information and Comm. Technologies and Development. 2-12.

[15] M. Khalil, J. Qadir, O. Onireti, M. A. Imran, and S. Younis. 2017. Feasibility, architecture and cost considerations of using TVWS for rural Internet access in 5G. In IEEE Conf. on Innovations in Clouds, Internet and Networks (ICIN). 23-30.

[16] D. Li and W. G. Halfond. 2014. An investigation into energy-saving programming practices for android smartphone app development. In Proceedings of the 3rd International Workshop on Green and Sustainable Software. ACM, 46-53.

[17] Y. C. Liang, A. T. Hoang, and H. H. Chen. 2008. Cognitive radio on TV bands: a new approach to provide wireless connectivity for rural areas. IEEE Wireless Communications 15, 3 (2008).

[18] H. Mehendale, A. Paranjpe, and S. Vempala. 2011. Lifenet: a flexible ad hoc networking solution for transient environments. In ACM SIGCOMM. 446-447.

[19] R. R. Mohassel, A. Fung, F. Mohammadi, and K. Raahemifar. 2014. A survey on advanced metering infrastructure. International fournal of Electrical Power \& Energy Systems 63 (2014), 473-484.

[20] [Online]. [n. d.]. FCC Report, "3.5Ghz CBRS: Report And Order and Second Further Notice Of Proposed Rulemaking.

[21] [Online]. [n. d.]. "http://www.rtl-sdr.com/rtl-sdr-wide-spectrum-analyzer/.

[22] [Online]. 2015. FCC, Second Memorandum Opinion and Order, ET Docket No FCC 10-174, September 2010.

[23] [Online]. 2015. Project Loon, www.google.com/loon.

[24] [Online]. 2017. 5G Americas White Paper on 5G Spectrum RecommendationsApril 2017. (2017). Last accessed on January 1, 2018.

[25] [Online], title="https://gsacom.com/5g-spectrum-bands/". [n. d.]

[26] A. Pentland, R. Fletcher, and A. Hasson. 2004. Daknet: Rethinking connectivity in developing nations. Computer 37, 1 (2004), 78-83.

[27] J. Ren, Y. Zhang, N. Zhang, D. Zhang, and X. Shen. 2016. Dynamic channel access to improve energy efficiency in cognitive radio sensor networks. IEEE Transactions on Wireless Communications 15, 5 (2016), 3143-3156.

[28] S. Saha, S. Nandi, P. S. Paul, V. K. Shah, A. Roy, and S. K. Das. 2015. Designing delay constrained hybrid ad hoc network infrastructure for post-disaster communication. Ad Hoc Networks 25 (2015), 406-429.

[29] A. Seth, D. Kroeker, M. Zaharia, S. Guo, and S. Keshav. 2006. Low-cost communication for rural internet kiosks using mechanical backhaul. In Proceedings of the 12th ACM int'l conf. on Mobile computing and networking. 334-345.

[30] H. Shokri-Ghadikolaei, I. Glaropoulos, V. Fodor, C. Fischione, and A. Ephremides. 2015. Green sensing and access: energy-throughput trade-offs in cognitive networking. IEEE Communications Magazine 53, 11 (2015), 199-207.

[31] K. D. Singh, P. Rawat, and J. M. Bonnin. 2014. Cognitive radio for vehicular ad hoc networks (CR-VANETs): approaches and challenges. EURASIP journal on wireless communications and networking 2014, 1 (2014), 49.

[32] P. Surampudi and S. Mohanty. 2011. LTE-advanced in white space: A complementary technology. Radisys White Paper (2011).

[33] R. H Tehrani, S. Vahid, D. Triantafyllopoulou, H. Lee, and K. Moessner. 2016. Licensed spectrum sharing schemes for mobile operators: A survey and outlook. IEEE Communications Surveys \& Tutorials 18, 4 (2016), 2591-2623.

[34] F. Theoleyre, T. Watteyne, G. Bianchi, G. Tuna, V. C. Gungor, and A. Pang. 2015. Networking and communications for smart cities special issue editorial. Computer Communications 58, 0 (2015), 1-3.

[35] X. Wu, S. Subramanian, R. Guha, R. G. White, J. Li, K. W. Lu, A. Bucceri, and T. Zhang. 2013. Vehicular communications using DSRC: challenges, enhancements, and evolution. IEEE fournal on Selected Areas in Comm. 31, 9 (2013), 399-408. 